\title{
PROJETO DE INTERFACE COLABORATIVA PARA SOFTWARE DE DESENVOLVIMENTO DE ONTOLOGIAS
}

\author{
Egon Sewald Junior \\ Instituto Federal de Santa \\ Catarina \\ Florianópolis, SC \\ egon.junior@ifsc.edu.br
}

\author{
Aires José Rover \\ Universidade Federal de Santa \\ Catarina \\ Florianópolis, SC \\ aires.rover@ccj.ufsc.br
}

\author{
Helio A. Ferenhof \\ Universidade Federal de Santa \\ Catarina \\ Florianópolis, SC \\ helio.ferenhof@ufsc.br
}

\section{RESUMO}

Esta pesquisa justifica-se por envolver o uso de tecnologia de informação e sistemas de conhecimento para apoiar a tomada de decisão e melhorar o desempenho da justiça brasileira. Considerando a formalização do direito na forma de documentos com bases textuais, o uso de sistemas de conhecimento depende da codificação do conhecimento linguístico que, nesses documentos, não é estruturado. Essa tarefa de codificação do conhecimento envolve recursos complexos, em grande número. Uma pesquisa-ação foi realizada e os resultados culminaram no desenvolvimento de projeto de interface que permite a execução das duas tarefas (construção de documentos e codificação de conhecimento). $\mathrm{O}$ projeto foi desenvolvido utilizando conceitos de priorização da experiência do usuário e buscando a possibilidade de colaboração entre usuários para reduzir a necessidade de recursos, aproveitando aqueles que constroem documentos com conhecimento linguístico. A validação foi realizada com usuários judiciais. $\mathrm{O}$ resultado da pesquisa é o design de interface para o sistema de conhecimento.

\section{Palavras-chaves}

Sistemas de Conhecimento; Ontologia; Colaboração; Interface com Usuário; User Experience

\section{ACM Classification Keywords}

H.5.2. Information interfaces and presentation (e.g., HCI): User Interfaces; I.7.1 Document and Text Processing: Document and Text Editing

\section{INTRODUÇÃO}

A República Federativa do Brasil é organizada a partir de três poderes: Executivo, Legislativo e Judiciário. A função de zelar a constituição e suas leis e manter o estado de direito, é de responsabilidade do poder judiciário, julgando conflitos de interesses, mantendo a ordem social. Para que

Permission to make digital or hard copies of all or part of this work for personal or classroom use is granted without fee provided that copies are not made or distributed for profit or commercial advantage and that copies bear this notice and the full citation on the first page. Copyrights for components of this work owned by others than the author(s) must be honored. Abstracting with credit is permitted. To copy otherwise, or republish, to post on servers or to redistribute to lists, requires prior specific permission and/or a fee. Copyright 2018 SBC.

IHC 2018, Anais Estendidos do XVII Simpósio Brasileiro sobre Fatores Humanos em Sistemas Computacionais

Outubro 22-26, 2018, Belém, Brasil

IHC na Prática isso possa ser possível, a atividade judiciária deve funcionar com desempenho suficiente a garantir tal ordem. Em busca da celeridade, o Poder Judiciário - assim como os demais poderes públicos - buscam a aplicação das técnicas de apoio à decisão, aplicadas de forma mais forte na administração empresarial, de modo a criar soluções que diminuam o tempo do processo no que tange a decisão, sentença ou similar.

Entre as tarefas intensivas em conhecimento na atividade judicante, a elaboração de sentenças e outras peças processuais, executada por operadores do direito, exige um grande tempo de pesquisa, buscando interpretações das leis, comparando com casos antigos similares ou mesmo retornando documentos que auxiliem o convencimento dos operadores, como é apontado por Sewald Junior [1]. Ainda, apresenta ainda que ferramentas de Engenharia de Conhecimento, como a busca semântica podem ser aplicadas para aumentar assertividade e relevância dos documentos resultantes da pesquisa e consequente diminuição do tempo total de busca.

Para o funcionamento dessas soluções, o conhecimento deve ser explicitado e codificado, com o uso de ontologias, por exemplo. A tarefa de explicitação do conhecimento em métodos manuais, ou seja, com a participação de especialistas de domínio e engenheiros de conhecimento, mostra-se de grande complexidade, exigindo um grande conjunto de recursos humanos e financeiros e, apesar do direito representar um dos principais motivos de desenvolvimento de ontologias, estas não alcançam a completude e complexidade do direito como um todo; e que os métodos automáticos, sobretudos os estatísticos, geram muitas relações irrelevantes e não é capaz de descrever ou conceituar os termos de forma suficiente.

\section{METODOLOGIA}

O objetivo deste trabalho e sua contribuição é a proposição de um projeto de interface colaborativa para representação do conhecimento judicial, com base no desenvolvimento de ontologias por grupo de especialistas de domínio de direito.

Foi, para o desenvolvimento deste trabalho, realizada uma busca sistemática de literatura com objetivo de gerar subsídios para definição do problema, e da composição dos pressupostos para o desenvolvimento de um projeto de interface. Para tal, foi aplicado o método Systematic Search 
Flow [2, 3]. 'No dia 25 de janeiro de 2017 a partir da expressão "("Collaborat*" AND "interface*" AND "user experience" )" na base Scopus, quando foram encontrados 76 registros. Forma utilizadas, como critério de exclusão, três passos: (1) eliminação de artigos sem relação com a pergunta de pesquisa a partir da leitura de título, resumo e palavras-chaves, resultando 25 artigos. Ainda, deste grupo, seis trabalhos não puderam ser acessados, impedindo essa leitura, totalizando, neste momento, 19 trabalhos; (2) repetição, da estratégia, a partir da leitura da integra dos artigos, resultando deste processo, 6 trabalhos; (3) integração de leituras anteriores a pesquisa, por parte do autor, bem como os textos acessíveis a ele, considerando indicações e trabalhos anteriores apresentados ao programa (teses e dissertações) - e textos de fundamentação a estes são integrados em temas laterais, quando encontradas lacunas a partir da revisão sistemática (considerando que a interseção de temas da pergunta de pesquisa pode limitar os resultados) na definição de temas periféricos à tese e quando possível acesso ao texto original. Também são buscados textos originais utilizados (citados) nos trabalhos resultantes da revisão sistemática de literatura, quando da existência de dúvidas quanto ao entendimento do conceito abordado e citado.

Com base nestes construtos gerados, uma pesquisa-ação, com a participação dos autores, resultando no desenvolvimento do projeto de interface, que foi verificado através de entrevistas a operadores do direito acessíveis ao autor, colaboradores de tribunais de justiça comum e do trabalho com atuação no estão de Santa Catarina.

\section{PROJETO DE INTERFACE COLABORATIVA}

Esta seção do artigo apresenta os fundamentos teóricos a partir da revisão sistemática de literatura, o projeto de interface resultante desses construtos e narrar o processo a verificação da aplicabilidade.

\subsection{Pressupostos teóricos}

Para o desenvolvimento de sistemas de conhecimento, o domínio de aplicação deve ser modelado e armazenado, possibilitando seu uso e reuso, definindo o vocabulário e os diferentes níveis de formalidade [4]. Neste contexto, encontramos as ontologias. Uma ontologia define os termos básicos e relacões compreendidos no vocabulário de uma área, bem como a combinação de termos e relações que definem a extensão do vocabulário [5] e denotam sistemas conceituais utilizados como veículos promotores do compartilhamento e reutilização do conhecimento [6].

Entre as principais razões para se desenvolver uma ontologia, destaca-se o compartilhamento de entendimento comum da estrutura da informação entre pessoas ou softwares agentes, possibilitando seu armazenamento, reuso e transmissão, com utilização do conceito de classes, relações, atributos, etc [7]. Desta forma, os conceitos e termos utilizados por um usuário poderiam ser entendidos por um sistema, e também o contrário, em suas diversas relações: hierárquicas, genéricas, associativas, entre outras.
Vários trabalhos utilizam as ontologias para auxiliar na identificação de conceitos e entidades relacionados com as sentenças, como, por exemplo, Penãlver-Martinez et al. [4], que define que uma mesma sentença pode ter diferentes significados / opiniões.

Com relação a construção da ontologia, Casanovas et al. definem o conceito de uma metodologia de engenharia de ontologia [8]: Uma metodologia de engenharia de ontologia pode ser definida como um conjunto organizado de procedimentos e diretrizes documentados para uma ou mais fases do ciclo de vida da ontologia, como análise ou design.

Os documentos jurídicos contêm conhecimento em forma de argumentos e é necessário distinguir entre argumento como processo e procedimento, da qual chama de dialético, e argumento como produto chamado não-dialético [9]. Este trabalho aponta que esta distinção serve para fins úteis para a engenharia do conhecimento na medida em que motivou o desenvolvimento de uma estrutura de representação do conhecimento que separe claramente as duas perspectivas. É descrita uma estrutura para a engenharia do conhecimento que suporta a perspectiva não-dialética expressivamente Zarri [10] aponta que documentos legais são estruturados a partir de conhecimentos narrativos. Observa-se que, durante o desenvolvimento de documentos, tarefa realizada por especialista da área do direito, os termos utilizados definem o entendimento do criador quanto ao seu significado, gerando ambiguidades apenas no processo de leitura e interpretação. $\mathrm{O}$ conhecimento aplicado no momento de geração do documento não é ambíguo, mas a sua leitura e ambiguidade de interpretação pode dificultar o entendimento do conceito utilizado.

Uma interface é o mecanismo utilizado para proporcionar interação entre dois entes (humano-computador, humanohumano, computador-computador etc) [11]. Assim, sendo, os conceitos de usabilidade e de experiência do usuário devem aplicar uma visão sistêmica, com base nas atividades e na interação entre os usuários e o sistema. Relacionado a sentimento do usuário, quanto ao uso de um sistema interativo, a experiência do Usuário (UX - User eXperience), centrada no usuário deve ser utilizada para o desenvolvimento.

Steves, Ranganathan e Morse [12] apresentam o uso de anotações, afirmando que pode ser adaptado para a execução de tarefas colaborativas. Apresenta a preocupação de, considerando execução com distância temporal entre os participantes, ferramentas assíncronas (para anotação) devem ser alinhadas com outras síncronas (para discussão), apesar de apresentar interfaces não aplicáveis ao projeto.

O trabalho de Gatto e Pittarello [13] contribui com um exemplo de interface para a anotação semântica, apesar do conceito de colaboração do autor esteja distante daquele de cooperação, necessário para o desenvolvimento do modelo de aplicação para suportar o projeto proposto. O uso de ontologia de anotações (Open Annotation Collaboration - 
OAC), apresentado no trabalho de Grassi, Morbidoni e Nucci [14] , mostra uma saída viável para a inserção/manutenção e compartilhamento de conceitos. A forma de compartilhamento (necessidade de escolha de compartilhamento) pode não ser útil para o projeto e não deverá ser aplicado. Contudo, o formato de anotação, populando uma ontologia, ajudará a identificar as palavrascandidatas. O uso de anotações para colaboração de uma tarefa (avaliação de livros virtuais - e-book), também é apresentado no trabalho de Yoo e Kim [15].

Partindo a limitação na formação da equipe de especialistas de domínio, para a construção da ontologia, é necessário cuidado no desenvolvimento de interface de interação que possibilite trabalho cotidiano do usuário (desenvolvimento de peças jurídicas). Considerando que a explicitação de conhecimentos jurídicos e seus conceitos no momento de sua utilização (criação de documentos jurídicos) possibilita a definição de uma ontologia jurídica não ambígua. A definição de interface de uma aplicação deve possibilitar o desenvolvimento destes documentos, a recomendação de palavras candidatas e a possibilidade de colaboração, por meio da avaliação (feedback / filtragem automática) reforçando ou inibindo recomendações de candidatas.

$\mathrm{O}$ projeto foi dividido, considerando o usuário e agrupamento de funcionalidades, em três módulos: (1) Módulo de Construção de Documentos Judiciais e Anotação; (2) Módulo de Decisão Cooperativa das Palavras Candidatas; e (3) Módulo de Inserção Manual na Ontologia, este último utilizado pelo Engenheiro de Conhecimento, enquanto os dois primeiros são utilizados pelo Especialista de Domínio.

\subsection{Módulo de Construção de Documentos Judiciais e Anotação}

A principal preocupação deste módulo é possibilitar a anotar o documento para identificação de palavras ou termos candidatos sem incremento impacto significativo de esforço, durante a elaboração de peças / documentos.

Após sua identificação, pode escolher iniciar um novo projeto (peça/ documento), continuar existente (realizar manutenção do documento e seu texto ou continuar tarefa de anotação) de sua autoria, ou de autoria outro usuário, desde que seja compartilhado a ele. Após a seleção do projeto, é aberto editor de texto, onde o usuário deve construir (gerar) suas peças judiciais e anotar o texto, podendo para isso, obter sugestões (quando a aplicação analisa, com base nas palavras já definidas no texto) e apresenta-las ao usuário, em uma forma de recomendação.

A Figura 1 apresenta uma visão geral do editor de texto, com os termos sem anotação marcados em amarelo. $\mathrm{O}$ usuário deve, a partir das recomendações ou de criação de nova candidata, realizar a anotação do documento, de forma a identificar palavras ou termos que são candidatas a ser incorporadas na ontologia. Tais palavras podem representar conceitos de classes, instâncias destas ou ainda, descrever relações entre instâncias e classes (para definição da ontologia, as relações entre instâncias são abstraídas, de forma a obter relacionamento entre classes destes termos).

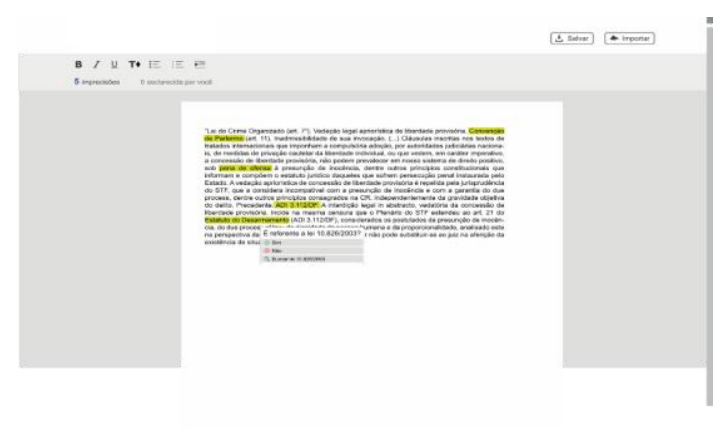

Figura 1 Protótipo de Interface - Sugestões com base nas candidatas existentes ou ontologia criada. Fonte: Elaborado pelo Autor

As palavras não anotadas podem receber recomendações a partir de palavras ou termos candidatos constantes da base e aqueles que já foram inseridos na ontologia; ou levar a sugestão de nova candidata, conforme apresentado na Figura 2.

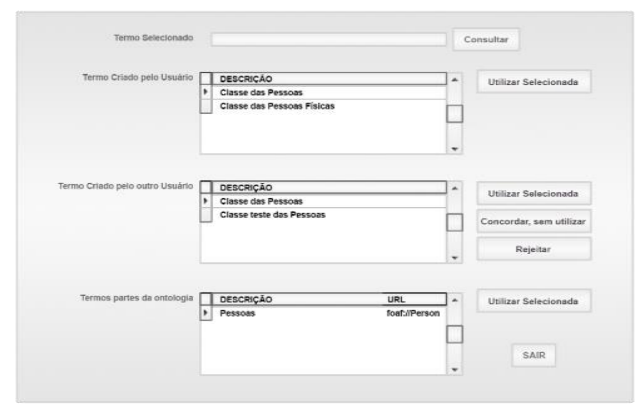

Figura 2 Protótipo de Interface - Sugestões de nova candidata. Fonte: Elaborado pelo Autor

O resultado esperado, com o uso deste módulo, é a possiblidade de anotação do documento (durante ou posterior ao sua criação), com a identificação de candidatas, sugerindo e/ou opinando e alimentando os indicadores de controle (filtragem colaborativa e reputação), considerando possibilidade de não existência de uma coordenação formal, sendo substituída por tais indicadores e gestão de papéis.

\subsection{Módulo de Decisão Colaborativa e Busca por Consensos das Palavras Candidatas}

Considerando a ambiguidade do direito, é possível que uma palavra ou termo possam ter significados diferentes, com base no contexto; isso, portanto, sugere que são dois ou mais conceitos distintos (e existir duas ou mais candidatas). Porém, também é possível o entendimento diferente sobre o mesmo conceito, em mesmo domínio (interpretação ou diferente definição por diferentes usuários). Nestes casos onde existem duas ou mais sugestões para o mesmo domínio, o Engenheiro de Ontologia, ao identificar, deve solicitar busca pelo consenso, em uma decisão cooperada. 
A Figura 3 apresenta interface gráfica para busca de consenso:

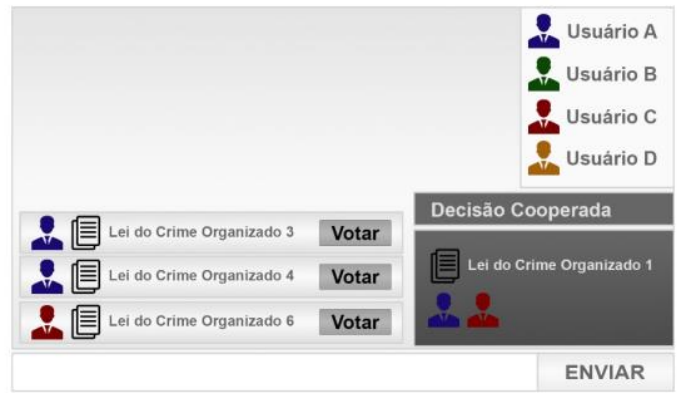

Figura 3 Protótipo de Interface - Decisão cooperativa e consensual - Chat e votação. Fonte: Elaborado pelo Autor

Tal interface permite a conversa entre os especialistas de domínio (operadores do direito, usuários do sistema de conhecimento), bem com sugestão de um conceito, votação nos conceitos sugeridos e, ao chegar a uma solução de consenso, incrementar o indicador de filtragem colaborativa como base nos pesos dos usuários (somados) que aceitaram, comumente, a candidata.

\subsection{Módulo de Inserção Manual na Ontologia}

Em grande parte das metodologias de aquisição de conhecimento que se apresentam como colaborativas, o engenheiro de ontologia tem a premissa de proceder a inserção da candidata na ontologia. De forma a manter essa prerrogativa do engenheiro de ontologia, é sugerido módulo de inserção manual na ontologia onde, ao observar que conceito é relevante e pode servir como termo compartilhado, promove sua inclusão.

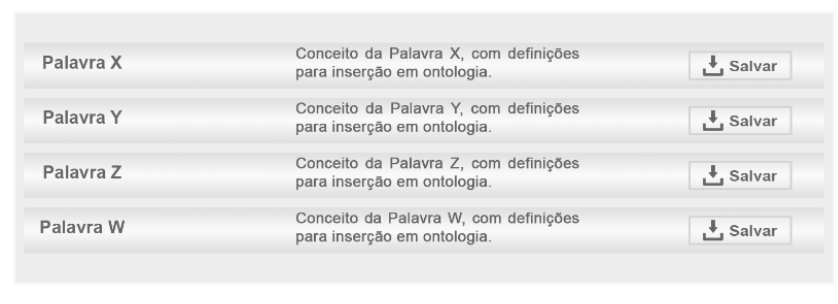

Figura 4 Protótipo de Interface - Inserção manual na ontologia. Fonte: Elaborado pelo Autor

A Figura 4 apresenta protótipo de interface com as sugestões acerca de uma mesma palavra ou termo, onde o engenheiro de ontologia pode salvar (inserir na base) ou refutar permanentemente.

Ainda, sugere-se que ao apresentar as candidatas, o engenheiro de ontologia possa observar o índice de filtragem colaborativa e a reputação do usuário que a sugeriu, como ferramenta para auxiliar a tomada de decisão. Pode, ainda, tomar essa decisão com apoio de um especialista (ou um grupo de especialistas) de domínio.

\subsection{Verificação com usuário}

Foram realizadas apresentações a juízes e desembargadores (justiça comum e trabalhista) e assessores, ou seja, possíveis usuários da solução baseada na proposta de interface gerada em tribunais de atuação no estado de Santa Catarina. A amostragem (12) é insuficiente para geração de estatísticas confiáveis quanto a validação, mas considerada suficiente pelos autores para a verificação da proposta.

Além do projeto de interface, foi demonstrado um possível resultado objetivado (ontologia) em outra área de interesse e sua aplicabilidade na busca semântica de documentos, e foi dada a palavra ao entrevistado para críticas, sugestões e percepção de aplicabilidade.

Em todos os casos, os possíveis usuários definiram que o ganho na seleção de documentos é muito útil no desenvolvimento de documentos jurídicos; que, apesar de um trabalho que diminui o desempenho, a percepção é de que o tempo total seria menor e, assim, que o software gerado a partir deste projeto seria aplicável.

Ainda, que o projeto apresentado, usando um mesmo ponto inicial para o desenvolvimento de documentos jurídicos e para anotação e consequente criação da ontologia representam uma experiência geral útil. A melhoria da seleção da classe ou da classe ancestral (no caso de criação de subclasse) utilizando uma árvore hierárquica foi sugerida, devendo ser incorporada em novos trabalhos, sobretudo no desenvolvimento do sistema de conhecimento.

\section{CONCLUSÕES}

Este trabalho demonstra um projeto de interação do usuário que permite a execução das duas tarefas de forma integrada, com a identificação de termos e definição colaborativa dos termos que farão parte da ontologia de domínio, aplicáveis em sistemas de conhecimento que visam apoiar a decisão. e tornar possível melhorar o desempenho das atividades judiciais. $\mathrm{O}$ projeto de interface com o usuário final levou em consideração a experiência do usuário, promovendo a percepção de usabilidade e possibilitando o uso de recursos humanos e computacionais, possibilitando ganhos com o uso desse conhecimento codificado e explicitados em outras soluções de software. A validação junto aos usuários demonstrou tal percepção, corroborando com a possibilidade de aplicação de design de interface no desenvolvimento de sistemas de conhecimento.

\section{AGRADECIMENTOS}

Os autores agradecem o Conselho Nacional de Justiça (CNJ) e (CAPES) pelo apoio financeiro para a realização da pesquisa; aos Tribunais de Justiça de Santa Catariana e Regional do Trabalho da $12^{\mathrm{a}}$ região pelo acesso aos seus colaboradores, necessários para entendimento da tarefa de desenvolvimento de documentos jurídicos.

\section{REFERÊNCIAS}

1. SEWALD JUNIOR, E., Modelagem de Sistema de Conhecimento para Apoio a Decisão Sentencial na Justiça Estadual, in Engenharia e Gestão do Conhecimento. 2012, Universidade Federal de Santa Catarina: Florianópolis. p. 159. 
2. FERENHOF, H.A. and R.F. FERNANDES, Revista ACB: Biblioteconomia em Santa Catarina. Revista ACB: Biblioteconomia em Santa Catarina, 2016. v. 21(n. 3): p. 550-563.

3. FERENHOF, H.A. and R.F. FERNANDES. Passos para construção da Revisão Sistemática e Bibliometria. v. 3.02. 2014 09 JUL 2017]; Available from: https://www.researchgate.net/publication/261322764_P asso-apasso_para_construcao_da_Revisao_Sistematica_e_Bi bliometria_Utilizando_a_ferramenta_EndnoteR.

4. PEÑALVER-MARTINEZ, I., et al., Feature-based opinion mining through ontologies. Expert Systems with Applications, 2014. 41(13): p. 5995-6008.

5. Neches, R., et al., Enabling Technology for Knowledge Sharing. AI Magazine, 1991: p. 36-56.

6. BATRES, E.J.Q., et al., Uso de ontologias para a extração de informações em atos jurídicos em uma instituição pública. Encontros Bibli: revista eletrônica de biblioteconomia e ciência da informação, 2005. v. 10(n. 19).

7. Gomez-Perez, A., M. Fernandez-Lopez, and O. Corcho, Ontological Engineering: with examples from the areas of knowledge management, ecommerce and the Semantic Web. Book by Springer, 2004.

8. Casanovas, P., et al., OPJK and DILIGENT: ontology modeling in a distributed environment. Artificial Intelligence and Law, 2007. 15(2): p. 171-186.

9. Stranieri, A., J. Zeleznikow, and J. Yearwood, Argumentation structures that integrate dialectical and non-dialectical reasoning. Knowledge Engineering Review, 2001. 16(4): p. 331-348.

10. Zarri, G.P., Advanced computational reasoning based on the NKRL conceptual model. Expert Systems with Applications, 2013. 40(8): p. 2872-2888.

11. GALITZ, W.O., The Essential Guide to User Interface Design. 2 ed. 2002, Estados Unidos da América: Wiley Computer Publishing. 786.

12. Steves, M.P., M. Ranganathan, and E. Morse, SMAT: Synchronous Multimedia and Annotation Tool. Proceedings of the Hawaii International Conference on System Sciences, 2001: p. 267.

13. Gatto, I. and F. Pittarello, Creating Web3D educational stories from crowdsourced annotations. Journal of Visual Languages and Computing, 2014. 25(6): p. 808817.

14. Grassi, M., C. Morbidoni, and M. Nucci, $A$ Collaborative Video Annotation System Based on Semantic Web Technologies. Cognitive Computation, 2012. 4(4): p. 497-514.

15. Yoo, H.S. and S.W. Kim, ESOTAG: E-book evolution using collaborative social tagging by readers. Multimedia Tools and Applications, 2016. 75(20): p. 12795-12813. 